\title{
Frequency Estimation in OFDM Direct-Conversion Receivers Using a Repeated Preamble
}

\author{
Antonio A. D'Amico, Leonardo Marchetti, Michele Morelli Senior Member, IEEE and Marco Moretti \\ Member, IEEE
}

\begin{abstract}
This paper investigates the problem of carrier frequency offset (CFO) recovery in an OFDM receiver affected by frequency-selective in-phase/quadrature (I/Q) imbalances. The analysis is based on maximum-likelihood (ML) methods and relies on the transmission of a training preamble with a repetitive structure in the time domain. After assessing the accuracy of the conventional ML (CML) scheme in a scenario characterized by I/Q impairments, we review the joint ML (JML) estimator of all unknown parameters and evaluate its theoretical performance. In order to improve the estimation accuracy, we also present a novel CFO recovery method that exploits some side-information about the signal-to-interference ratio. It turns out that both CML and JML can be derived from this scheme by properly adjusting the value of a design parameter. The accuracy of the investigated methods are compared with the relevant Cramer-Rao bound. Our results can be used to check whether conventional CFO recovery algorithms can work properly or not in the presence of I/Q imbalances and also to evaluate the potential gain attainable by more sophisticated schemes.
\end{abstract}

Index Terms-Frequency recovery, OFDM, direct-conversion receiver, I/Q imbalance.

\section{INTRODUCTION}

In recent years, the combination of OFDM with the directconversion receiver (DCR) concept has attracted considerable attention [1]. In contrast to the classical superheterodyne architecture, in a DCR device the radio-frequency (RF) signal is down-converted to baseband without passing through any intermediate-frequency (IF) stage. On the one hand, this approach avoids the use of expensive image rejection filters and other off-chip components, with a remarkable advantage in terms of cost and circuit board size. On the other hand, a DCR front-end introduces some RF/analog imbalances arising from the use of in-phase/quadrature (I/Q) low-pass filters (LPFs) with mismatched frequency responses, and from local oscillator (LO) signals with unequal amplitudes and imperfect $90^{\circ}$ phase difference. Overall, I/Q non-idealities give rise to conjugate mirror-image interference on the down-converted signal, which can seriously degrade the system performance. An OFDM receiver also exhibits a remarkable sensitivity to the carrier frequency offset (CFO) between the received waveform and the LO signals, which originates interchannel interference (ICI) at the output of the discrete Fourier transform (DFT) unit.

An intense research activity has been recently devoted to the problem of CFO recovery in OFDM systems plagued by

The authors are with the Department of Information Engineering, University of Pisa (e-mail: \{antonio.damico, leonardo.marchetti, michele.morelli, marco.moretti\}@iet.unipi.it). frequency-selective I/Q imperfections. The methods presented in [2] and [3] exploit a dedicated training preamble (TP) composed of three repeated parts to retrieve the cosine of the normalized CFO. However, since the cosine is an even function of its argument, the frequency estimates are affected by an inherent sign ambiguity. In [4]-[6] the original preamble proposed in [2] is extended by a second part which is rotated by an artificial frequency shift before transmission. The resulting TP allows one to recover both the cosine and the sine of the CFO, which are eventually combined to get unambiguous estimates of the frequency offset. A similar approach is adopted in [7], where the sign ambiguity problem is fixed by rotating the repeated parts of the TP by a specified phase pattern. Albeit effective, all the aforementioned solutions cannot be applied to practical OFDM systems since they rely on suitably designed TPs that cannot be found in any commercial standard.

The schemes presented in [8]-[12] exploit the conventional repeated TP of the IEEE 802.11a WLAN standard. Specifically, in [8] the authors present a suitable matrix formulation of the received signal samples to derive novel sine and cosine-based CFO estimators, while the frequency-domain correlations of the TP are used in [9]. An alternative cosinebased estimator is derived in [10] using a general relation among three arbitrary TP segments, while rotational invariance techniques (ESPRIT) [13] are applied in [11]. Finally, an iterative interference-cancellation approach is presented in [12] by resorting to the space-alternating generalized expectationmaximization (SAGE) algorithm [14].

The common idea behind all the aforementioned schemes is that conventional CFO estimators cannot work properly when applied to a DCR architecture. However, so far only numerical measurements and heuristic arguments have been used to support such an established belief, while any solid theoretical analysis is still missing. This paper tries to fill such a gap by providing a theoretical investigation of the CFO recovery problem in an OFDM receiver affected by frequency-selective I/Q imbalance. In doing so, we adopt a maximum-likelihood (ML) approach and consider a burstmode transmission wherein each frame is preceded by the conventional repeated TP. Our goal is to provide answers to the following key questions: $i$ ) To which extent can conventional CFO recovery schemes perform satisfactorily in the presence of RF imperfections? ii) How do CFO recovery schemes devised for DCR architectures compare with conventional methods that ignore the presence of I/Q imbalances? iii) Is it possible to design more sophisticated algorithms to improve the accuracy of available methods? iv) Can such improved 
performance be achieved with a tolerable increase of the system complexity?

In order to address question $i$ ), we begin our study by reviewing the classical ML (CML) frequency estimator presented in [15] and analytically assessing its accuracy in the presence of I/Q imbalances. This analysis, which is not available in the literature, is important for establishing the price (in terms of estimation accuracy) that must be paid when applying CML in an I/Q imbalance scenario. Next, we assess the theoretical performance of the algorithm presented in [7] for the joint ML (JML) estimation of the CFO, the channeldistorted TP and its mirror image. Such an analysis is not available in [7] and provides an answer to question $i i$ ). As we shall see, JML is very sensitive to the magnitude of the CFO value and fails whenever the CFO becomes vanishingly small. Motivated by such a result, we move to question $i$ ii) and derive a novel ML-based estimator of all the unknown parameters which exploits some side information about the average signalto-image ratio (SIR). Such an estimator can be interpreted as an extension of both CML and JML since the latter schemes are obtained from the former by simply adjusting a design parameter. Compared to CML and JML, the new estimator provides improved accuracy at the price of a certain increase of the computational load. The complexity analysis of CML, JML and CJML is eventually used to answer question $i v$ ). A last contribution is the derivation of the Cramer-Rao bound (CRB) for $\mathrm{CFO}$ recovery in the presence of I/Q imbalance using the true noise statistics. This result can be used to check whether the approximated bound derived under the traditional white Gaussian noise (WGN) assumption deviates substantially or not from the true CRB.

The rest of the paper is organized as follows. Next section illustrates the DCR architecture and introduces the signal model. In Sects. III and IV we review the CML and JML, respectively, while the novel CFO estimator exploiting SIR information is derived in Sect. V. We provide the CRB analysis in Sect. VI and discuss simulation results in Sect. VII. Finally, some conclusions are drawn in Sect. VIII.

Notation: Matrices and vectors are denoted by boldface letters, with $\mathbf{I}_{N}$ and $\mathbf{0}$ being the identity matrix of order $N$ and the null vector, respectively. $\mathbf{A}=\operatorname{diag}\{a(n) ; n=$ $1,2, \ldots, N\}$ denotes an $N \times N$ diagonal matrix with entries $a(n)$ along its main diagonal, while $\mathbf{B}^{-1}$ is the inverse of a square matrix $\mathbf{B}$. We use $\mathrm{E}\{\cdot\},(\cdot)^{*},(\cdot)^{T}$ and $(\cdot)^{H}$ for expectation, complex conjugation, transposition and Hermitian transposition, respectively. The notation $\arg \{\cdot\}$ stands for the argument of a complex-valued quantity, $|\cdot|$ represents the corresponding modulus, while the real and imaginary parts are expressed by $\operatorname{Re}(\cdot)$ and $\operatorname{Im}(\cdot)$, respectively. Finally, we denote by $\tilde{\lambda}$ a trial value of an unknown parameter $\lambda$.

\section{Signal Model in The PRESEncE of I/Q IMBALANCE}

\section{A. Direct conversion receiver}

Fig. 1 illustrates the basic DCR architecture in the presence of I/Q imbalances. The latter originate from I/Q filters with mismatched impulse responses $g_{I}(t)$ and $g_{Q}(t)$, as well as from LO signals with an amplitude imbalance $\alpha$ and a phase

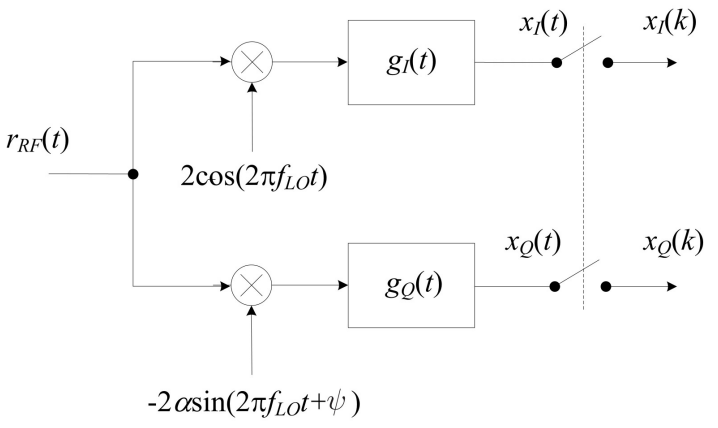

Fig. 1. Basic architecture of a direct-conversion receiver.

error $\psi$. We call $s(t)$ and $v(t)$ the baseband representations of the transmitted signal and propagation channel, respectively. Then, denoting by $r(t)$ the complex envelope of the received waveform $r_{R F}(t)$ with respect to the carrier frequency $f_{0}$, we have $r(t)=s(t) \otimes v(t)+n(t)$, with $n(t)$ being circularly symmetric AWGN with two-sided power spectral density $2 N_{0}$. From the analysis in [16], the down-converted baseband signal $x(t)=x_{I}(t)+j x_{Q}(t)$ can be written as

$x(t)=e^{j 2 \pi \Delta f t}[s(t) \otimes h(t)]+e^{-j 2 \pi \Delta f t}\left[s^{*}(t) \otimes q(t)\right]+w(t)$

where $\Delta f=f_{0}-f_{L O}$ is the offset between the carrier and LO frequencies, while the impulse responses $h(t)$ and $q(t)$ are defined as

$$
\begin{aligned}
h(t) & =v(t) \otimes\left[p_{+}(t) e^{-j 2 \pi \Delta f t}\right] \\
q(t) & =v^{*}(t) \otimes\left[p_{-}(t) e^{j 2 \pi \Delta f t}\right]
\end{aligned}
$$

with $p_{+}(t)=0.5 \cdot\left[g_{I}(t)+\alpha g_{Q}(t) e^{-j \psi}\right]$ and $p_{-}(t)=0.5$. $\left[g_{I}(t)-\alpha g_{Q}(t) e^{j \psi}\right]$. Finally, the noise term $w(t)$ is related to $n(t)$ by

$$
w(t)=n(t) e^{j 2 \pi \Delta f t} \otimes p_{+}(t)+n^{*}(t) e^{-j 2 \pi \Delta f t} \otimes p_{-}(t) .
$$

Letting $w(t)=w_{I}(t)+j w_{Q}(t)$, it follows that $w_{I}(t)$ and $w_{Q}(t)$ are zero-mean Gaussian processes with auto- and crosscorrelation functions

$$
\begin{gathered}
\mathrm{E}\left\{w_{I}(t) w_{I}(t+\tau)\right\}=N_{0}\left[g_{I}(\tau) \otimes g_{I}(-\tau)\right] \\
\mathrm{E}\left\{w_{Q}(t) w_{Q}(t+\tau)\right\}=\alpha^{2} N_{0}\left[g_{Q}(\tau) \otimes g_{Q}(-\tau)\right] \\
\mathrm{E}\left\{w_{I}(t) w_{Q}(t+\tau)\right\}=-\alpha N_{0} \sin \psi\left[g_{I}(\tau) \otimes g_{Q}(-\tau)\right] .
\end{gathered}
$$

Inspection of (4) reveals that $w(t)$ is not circularly symmetric as its real and imaginary components are generally crosscorrelated and have different auto-correlation functions.

\section{B. Signal model}

The investigated system is an OFDM burst-mode transceiver where each block has length $T$ and is preceded by a cyclic prefix (CP) to avoid interblock interference. We denote by $N$ the number of available subcarriers and by $1 / T$ the subcarrier spacing. As specified in [17], a TP is appended in front of each data frame to facilitate the synchronization task. In particular, we assume that the TP has a periodic structure in the timedomain and is composed by $M \geq 2$ identical segments [18], [19]. The basic segment comprises $P$ time-domain samples (with $P$ being a power of two) and is generated by feeding a 
sequence of pilot symbols $\mathbf{c}=[c(0), c(1), \ldots, c(P-1)]^{T}$ into a $P$-point inverse DFT unit. Hence, denoting by $s(k)$ the $k$ th sample of the TP, we have

$$
s(k)=\frac{1}{\sqrt{P}} \sum_{n=0}^{P-1} c(n) e^{j 2 \pi n k / P} \quad-N_{g} \leq k \leq M P-1
$$

where $N_{g}$ is the CP length normalized by the signaling period $T_{s}=T / N$.

After propagating through a multipath channel, the received signal $r_{R F}(t)$ is down-converted to baseband and sampled with period $T_{s}$ using the DCR architecture of Fig. 1. Then, samples belonging to the TP are arranged into $M$ vectors $\mathbf{x}_{m}=\left[x_{m}(0), x_{m}(1), \ldots, x_{m}(P-1)\right]^{T}(m=0,1, \ldots, M-$ $1)$, each of them having length $P$ and corresponding to a specific TP segment. According to (1), the $p$ th entry of $\mathbf{x}_{m}$ can be written as

$x_{m}(p)=e^{j[m-(M-1) / 2] \varphi} a(p)+e^{-j[m-(M-1) / 2] \varphi} b(p)+w_{m}(p)$

where $w_{m}(p)$ is the noise contribution and we have defined

$$
\varphi=\frac{2 \pi \nu}{Q}
$$

with $Q=N / P$ and $\nu \triangleq \Delta f \cdot T$ being the CFO normalized by the subcarrier spacing. Furthermore, $a(p)$ and $b(p)$ are given by

$$
\begin{gathered}
a(p)=e^{j(M-1) \varphi / 2} e^{j 2 \pi \nu p / N}[s(t) \otimes h(t)]_{t=p T_{s}} \\
b(p)=e^{-j(M-1) \varphi / 2} e^{-j 2 \pi \nu p / N}\left[s^{*}(t) \otimes q(t)\right]_{t=p T_{s}}
\end{gathered}
$$

where

$$
s(t)=\frac{1}{\sqrt{P}} \sum_{n=0}^{P-1} c(n) e^{j 2 \pi n Q t / T}
$$

is the transmitted TP. In writing (8) and (9), we have borne in mind that $[s(t) \otimes h(t)]_{t=p T_{s}}$ and $\left[s^{*}(t) \otimes q(t)\right]_{t=p T_{s}}$ are periodic in $p$ of period $P$ due to the repetitive TP structure.

To proceed further, we consider the following $M$-dimensional vectors

$\mathbf{x}(p)=\left[x_{0}(p), x_{1}(p), \ldots, x_{M-1}(p)\right]^{T} \quad p=0,1, \ldots, P-1$

where $\mathbf{x}(p)$ is obtained by collecting the $p$ th entry of $\left\{\mathbf{x}_{m}\right\}_{m=0}^{M-1}$. Hence, from (6) we get

$$
\mathbf{x}(p)=\mathbf{u}(\varphi) a(p)+\mathbf{u}(-\varphi) b(p)+\mathbf{w}(p)
$$

where $\mathbf{w}(p)=\left[w_{0}(p), w_{1}(p), \ldots, w_{M-1}(p)\right]^{T}$ is a zero-mean Gaussian vector and

$$
\mathbf{u}(\varphi)=e^{-j(M-1) \varphi / 2}\left[1, e^{j \varphi}, e^{j 2 \varphi}, \ldots, e^{j(M-1) \varphi}\right]^{T} .
$$

Inspection of (12) and (13) reveals that $\mathbf{x}(p)$ consists of two spectral lines $\mathbf{u}(\varphi)$ and $\mathbf{u}(-\varphi)$, symmetrically positioned around the origin and accounting for the direct signal and its mirror image, respectively. In the ensuing discussion, we investigate the ML estimation of the normalized CFO $\varphi$ in the presence of the nuisance vectors $\mathbf{a}=[a(0), a(1), \ldots, a(P-$ $1)]^{T}$ and $\mathbf{b}=[b(0), b(1), \ldots, b(P-1)]^{T}$. In particular, we begin by reviewing the CML estimator presented in [15], which assumes $\mathbf{b}=\mathbf{0}$, and evaluate its performance in the presence of I/Q imbalance. Next, we assess the accuracy of the JML algorithm proposed in [7], which jointly estimates $(\varphi, \mathbf{a}, \mathbf{b})$ without exploiting any side information about $\mathbf{b}$. Such theoretical analysis will be used to compare the accuracy of CML and JML in the presence of I/Q imbalance. Since the signal component is typically much stronger than its mirror image (i.e., $\|\mathbf{a}\| \gg\|\mathbf{b}\|)$, a novel ML estimator of $(\varphi, \mathbf{a}, \mathbf{b})$ is eventually derived by putting a constraint on the ratio $\|\mathbf{a}\|^{2} /\|\mathbf{b}\|^{2}$.

To make the analysis mathematically tractable, we model the noise term $w(t)$ as a zero-mean circularly-symmetric Gaussian (ZMCSG) complex random process. This amounts to saying that $\{\mathbf{w}(p) ; p=0,1, \ldots, P-1\}$ are statistically independent ZMCSG vectors with covariance matrix $\mathbf{K}_{w}=$ $\sigma_{w}^{2} \mathbf{I}_{M}$. Although this assumption holds true only in the case of a perfectly balanced DCR scheme, it has been largely adopted in the literature even in the presence of non-negligible RF imperfections [20]. In this work, the white noise assumption is employed only to derive the frequency estimation algorithms and for their performance analysis, while the true noise statistics shown in (4) are used in the numerical simulations and for the CRB evaluation.

\section{CFO ESTIMATION IN THE ABSENCE OF I/Q IMBALANCE}

\section{A. Estimator's design}

The CML is proposed in [15] for an OFDM receiver free from any RF imperfection. This scheme performs the joint ML estimation of $(\varphi, \mathbf{a})$ based on the following signal model

$$
\mathbf{x}(p)=\mathbf{u}(\varphi) a(p)+\mathbf{w}(p) \quad p=0,1, \ldots, P-1 .
$$

The log-likelihood function (LLF) is expressed by [21]

$$
\Lambda(\tilde{\varphi}, \tilde{\mathbf{a}})=-N \ln \left(\pi \sigma_{w}^{2}\right)-\frac{1}{\sigma_{w}^{2}} \sum_{p=0}^{P-1}\|\mathbf{x}(p)-\mathbf{u}(\tilde{\varphi}) \tilde{a}(p)\|^{2}
$$

and its maximization with respect to $(\tilde{\varphi}, \tilde{\mathbf{a}})$ leads to the following CFO estimate

$$
\hat{\varphi}_{C M L}=\arg \max _{\tilde{\varphi} \in[-\pi, \pi)}\left\{\Psi_{C M L}(\tilde{\varphi})\right\}
$$

where

$$
\Psi_{C M L}(\tilde{\varphi})=\sum_{p=0}^{P-1}\left|\mathbf{u}^{H}(\tilde{\varphi}) \mathbf{x}(p)\right|^{2} .
$$

Taking (11) and (13) into account, we may put the metric $\Psi_{C M L}(\tilde{\varphi})$ in the equivalent form

$$
\Psi_{C M L}(\tilde{\varphi})=\sum_{m=0}^{M-1} \sum_{k=0}^{M-1} \operatorname{Re}\left\{\chi_{C M L, m, k}(\tilde{\varphi}) \mathbf{x}_{m}^{H} \mathbf{x}_{k}\right\}
$$

with $\chi_{C M L, m, k}(\tilde{\varphi})=e^{j(m-k) \tilde{\varphi}}$. 


\section{B. Performance analysis}

Since the CML is derived under the simplifying assumption $\mathbf{b}=\mathbf{0}$, it is interesting to assess its accuracy in the presence of I/Q imbalance. For this purpose, we define the estimation error as $\varepsilon_{C M L}=\varphi-\hat{\varphi}_{C M L}$, and we analyse the CML performance assuming relatively small values of $\varepsilon_{C M L}$. Hence, following the approach outlined in [22], we get

$$
\begin{aligned}
\mathrm{E}\left\{\varepsilon_{C M L}\right\} & \simeq-\frac{\mathrm{E}\left\{\Psi_{C M L}^{\prime}(\varphi)\right\}}{\mathrm{E}\left\{\Psi_{C M L}^{\prime \prime}(\varphi)\right\}} \\
\mathrm{E}\left\{\varepsilon_{C M L}^{2}\right\} & \simeq \frac{\mathrm{E}\left\{\left[\Psi_{C M L}^{\prime}(\varphi)\right]^{2}\right\}}{\left[\mathrm{E}\left\{\Psi_{C M L}^{\prime \prime}(\varphi)\right\}\right]^{2}}
\end{aligned}
$$

where $\Psi_{C M L}^{\prime}(\varphi)$ and $\Psi_{C M L}^{\prime \prime}(\varphi)$ are the first and second order derivatives of $\Psi_{C M L}(\tilde{\varphi})$, respectively, evaluated at $\tilde{\varphi}=\varphi$. In Appendix A it is shown that

$$
\mathrm{E}\left\{\varepsilon_{C M L}\right\}=\frac{6}{M^{2}-1} \cdot \frac{q_{M}^{\prime}(\varphi)\left[\operatorname{Re}\left(\mathbf{a}^{H} \mathbf{b}\right)+q_{M}(\varphi)\|\mathbf{b}\|^{2}\right]}{\Omega_{M}(\varphi)}
$$

with

$$
q_{M}(\varphi)=\frac{\sin (M \varphi)}{M \sin \varphi}
$$

and

$$
\begin{aligned}
\Omega_{M}(\varphi) & =\|\mathbf{a}\|^{2}+\left[q_{M}(\varphi)-\gamma_{M}(\varphi)\right] \operatorname{Re}\left(\mathbf{a}^{H} \mathbf{b}\right) \\
& -\left[\beta_{M}(\varphi)+q_{M}(\varphi) \gamma_{M}(\varphi)\right]\|\mathbf{b}\|^{2} .
\end{aligned}
$$

In the above equation, the quantities $\beta_{M}(\varphi)$ and $\gamma_{M}(\varphi)$ are expressed by

$\beta_{M}(\varphi)=\frac{3}{M^{2}-1}\left[q_{M}^{\prime}(\varphi)\right]^{2}$ and $\gamma_{M}(\varphi)=\frac{3}{M^{2}-1} q_{M}^{\prime \prime}(\varphi)$

where $q_{M}^{\prime}(\varphi)$ and $q_{M}^{\prime \prime}(\varphi)$ are the first and second order derivatives of $q_{M}(\varphi)$, respectively. From (21)-(23) we see that $\hat{\varphi}_{C M L}$ is a biased estimate of $\varphi$. The only exceptions occur in the absence of I/Q imbalance or when $\varphi=0$, since in the latter case we have $q_{M}^{\prime}(\varphi)=0$.

In Appendix A we also evaluate the mean square estimation error (MSEE) of $\hat{\varphi}_{C M L}$, which is found to be

$$
\begin{aligned}
\mathrm{E}\left\{\varepsilon_{C M L}^{2}\right\}=\mathrm{E}^{2}\left\{\varepsilon_{C M L}\right\} & +\frac{6 \sigma_{w}^{2}}{M\left(M^{2}-1\right)} \cdot \frac{A_{M}(\varphi)}{\Omega_{M}^{2}(\varphi)} \\
& +\frac{6 P \sigma_{w}^{4}}{M^{2}\left(M^{2}-1\right)} \cdot \frac{1}{\Omega_{M}^{2}(\varphi)}
\end{aligned}
$$

with

$$
A_{M}(\varphi)=\|\mathbf{a}\|^{2}+2 q_{M}(\varphi) \operatorname{Re}\left(\mathbf{a}^{H} \mathbf{b}\right)+\left[\beta_{M}(\varphi)+q_{M}^{2}(\varphi)\right]\|\mathbf{b}\|^{2} .
$$

\section{Remarks}

i) Observing that $q_{M}(0)=1, \beta_{M}(0)=0$ and $\gamma_{M}(0)=-1$, for $\varphi=0$ we get $A_{M}(0)=\Omega_{M}(0)=\|\mathbf{a}+\mathbf{b}\|^{2}$ and (25) reduces to

$\left.\mathrm{E}\left\{\varepsilon_{C M L}^{2}\right\}\right|_{\varphi=0}=\frac{6 \sigma_{w}^{2}}{M\left(M^{2}-1\right)\|\mathbf{a}+\mathbf{b}\|^{2}}\left[1+\frac{P \sigma_{w}^{2}}{M\|\mathbf{a}+\mathbf{b}\|_{(27)}^{2}}\right]$. ii) In the absence of I/Q imbalance we have $A_{M}(\varphi)=$ $\Omega_{M}(\varphi)=\|\mathbf{a}\|^{2}$. In such a case, (25) becomes independent of $\varphi$ and takes the form

$$
\left.\mathrm{E}\left\{\varepsilon_{C M L}^{2}\right\}\right|_{\mathbf{b}=\mathbf{0}}=\frac{6 \sigma_{w}^{2}}{M\left(M^{2}-1\right)\|\mathbf{a}\|^{2}}\left(1+\frac{P \sigma_{w}^{2}}{M\|\mathbf{a}\|^{2}}\right)
$$

which further simplifies to

$$
\left.\mathrm{E}\left\{\varepsilon_{C M L}^{2}\right\}\right|_{\mathbf{b}=\mathbf{0},\|\mathbf{a}\|^{2} / \sigma_{w}^{2} \rightarrow \infty}=\frac{6 \sigma_{w}^{2}}{M\left(M^{2}-1\right)\|\mathbf{a}\|^{2}}
$$

at relatively high $\mathrm{SNR}$ values (i.e., for $\|\mathbf{a}\|^{2} / \sigma_{w}^{2} \rightarrow \infty$ ). It is worth noting that the right-hand side of (29) is the CRB for $\mathrm{CFO}$ estimation reported in [15]. This means that CML is asymptotically efficient when $\mathbf{b}=\mathbf{0}$.

\section{JOINT ML ESTIMATION OF THE UNKNOWN PARAMETERS}

\section{A. Estimator's design}

In this section we review the JML presented in [7], which aims at jointly estimating the unknown parameters $(\varphi, \mathbf{a}, \mathbf{b})$. After rewriting (12) as

$$
\mathbf{x}(p)=\mathbf{A}_{2}(\varphi) \boldsymbol{\theta}(p)+\mathbf{w}(p) \quad p=0,1, \ldots, P-1
$$

with $\mathbf{A}_{2}(\varphi)=[\mathbf{u}(\varphi) \mathbf{u}(-\varphi)]$ and $\boldsymbol{\theta}(p)=[a(p), b(p)]^{T}$, the LLF takes the form

$$
\Lambda_{2}(\tilde{\varphi}, \tilde{\boldsymbol{\theta}})=-N \ln \left(\pi \sigma_{w}^{2}\right)-\frac{1}{\sigma_{w}^{2}} \sum_{p=0}^{P-1}\left\|\mathbf{x}(p)-\mathbf{A}_{2}(\tilde{\varphi}) \tilde{\boldsymbol{\theta}}(p)\right\|^{2}
$$

where $\tilde{\boldsymbol{\theta}}(p) \triangleq[\tilde{a}(p), \tilde{b}(p)]^{T}$ and $\tilde{\boldsymbol{\theta}}=\{\tilde{\boldsymbol{\theta}}(0), \tilde{\boldsymbol{\theta}}(1), \ldots, \tilde{\boldsymbol{\theta}}(P-$ $1)\}$. The maximum of the LLF with respect to $\tilde{\boldsymbol{\theta}}(p)$ is attained at

$$
\hat{\boldsymbol{\theta}}(p ; \tilde{\varphi})=\left[\mathbf{A}_{2}^{H}(\tilde{\varphi}) \mathbf{A}_{2}(\tilde{\varphi})\right]^{-1} \mathbf{A}_{2}^{H}(\tilde{\varphi}) \mathbf{x}(p)
$$

which is next substituted into (31) in place of $\tilde{\boldsymbol{\theta}}(p)$, yielding the concentrated likelihood function

$$
\Lambda_{2}(\tilde{\varphi})=-N \ln \left(\pi \sigma_{w}^{2}\right)-\frac{1}{\sigma_{w}^{2}} \sum_{p=0}^{P-1} \mathbf{x}^{H}(p)\left[\mathbf{I}_{M}-\mathbf{C}_{2}(\tilde{\varphi})\right] \mathbf{x}(p)
$$

with $\mathbf{C}_{2}(\tilde{\varphi})=\mathbf{A}_{2}(\tilde{\varphi})\left[\mathbf{A}_{2}^{H}(\tilde{\varphi}) \mathbf{A}_{2}(\tilde{\varphi})\right]^{-1} \mathbf{A}_{2}^{H}(\tilde{\varphi})$. The $\mathrm{ML}$ estimate of $\varphi$ is eventually given by

$$
\hat{\varphi}_{J M L}=\arg \max _{\tilde{\varphi} \in[-\pi, \pi)}\left\{\Psi_{J M L}(\tilde{\varphi})\right\}
$$

where

$$
\Psi_{J M L}(\tilde{\varphi})=M \sum_{p=0}^{P-1} \mathbf{x}^{H}(p) \mathbf{C}_{2}(\tilde{\varphi}) \mathbf{x}(p) .
$$

After some manipulations, it is found that the metric $\Psi_{J M L}(\tilde{\varphi})$ can also be written as

$$
\Psi_{J M L}(\tilde{\varphi})=\sum_{m=0}^{M-1} \sum_{k=0}^{M-1} \operatorname{Re}\left\{\chi_{J M L, m, k}(\tilde{\varphi}) \mathbf{x}_{m}^{H} \mathbf{x}_{k}\right\}
$$

where

$\chi_{J M L, m, k}(\tilde{\varphi})=\frac{\cos [(m-k) \tilde{\varphi}]-q_{M}(\tilde{\varphi}) \cos [(m+k-M+1) \tilde{\varphi}]}{1-q_{M}^{2}(\tilde{\varphi})}$ 
and $q_{M}(\tilde{\varphi})$ is defined in (22).

It is worth noting that letting $M=2$ yields $\mathbf{C}_{2}(\tilde{\varphi})=\mathbf{I}_{2}$, which makes $\Psi_{J M L}(\tilde{\varphi})$ independent of $\tilde{\varphi}$. This amounts to saying that application of JML is possible only for $M \geq 3$. Furthermore, since $\Psi_{J M L}(\tilde{\varphi})$ is an even function of $\tilde{\varphi}$, it exhibits two global maxima symmetrically positioned around $\tilde{\varphi}=0$. This results into an ambiguity in the sign of $\hat{\varphi}_{J M L}$ which cannot be removed unless additional information is available. One possible solution relies on the fact that the useful signal component is typically much stronger than its mirror image. Hence, we suggest to consider the positive solution of (34), say $\hat{\varphi}_{J M L}^{+}$, and compute the estimates $\hat{\mathbf{a}}$ and $\hat{\mathbf{b}}$ from (32) after replacing $\tilde{\varphi}$ with $\hat{\varphi}_{J M L}^{+}$. Then, we set $\hat{\varphi}_{J M L}=\hat{\varphi}_{J M L}^{+}$if $\|\hat{\mathbf{a}}\|>\|\hat{\mathbf{b}}\|$, otherwise we choose $\hat{\varphi}_{J M L}=-\hat{\varphi}_{J M L}^{+}$.

\section{B. Performance analysis}

The accuracy of $\hat{\varphi}_{J M L}$ is assessed by applying the same methods used for $\hat{\varphi}_{C M L}$. Skipping the details, it is found that $\mathrm{E}\left\{\hat{\varphi}_{J M L}\right\}=\varphi$, thereby indicating that JML is unbiased. Furthermore, denoting by $\varepsilon_{J M L}=\varphi-\hat{\varphi}_{J M L}$ the estimation error, the MSEE turns out to be

$$
\begin{aligned}
\mathrm{E}\left\{\varepsilon_{J M L}^{2}\right\} & =\frac{6 \sigma_{w}^{2}\left[M\left(M^{2}-1\right)\right]^{-1}}{\left[\Gamma_{M, 1}(\varphi)\left(\|\mathbf{a}\|^{2}+\|\mathbf{b}\|^{2}\right)+2 \Gamma_{M, 2}(\varphi) \operatorname{Re}\left(\mathbf{a}^{H} \mathbf{b}\right)\right]} \\
& +\frac{12 P \sigma_{w}^{4} \Gamma_{M, 3}(\varphi)\left[M^{2}\left(M^{2}-1\right)\right]^{-1}}{\left[\Gamma_{M, 1}(\varphi)\left(\|\mathbf{a}\|^{2}+\|\mathbf{b}\|^{2}\right)+2 \Gamma_{M, 2}(\varphi) \operatorname{Re}\left(\mathbf{a}^{H} \mathbf{b}\right)\right]^{2}}
\end{aligned}
$$

where

$$
\begin{gathered}
\Gamma_{M, 1}(\varphi)=1-\frac{\beta_{M}(\varphi)}{1-q_{M}^{2}(\varphi)} \\
\Gamma_{M, 2}(\varphi)=\gamma_{M}(\varphi)+\frac{\beta_{M}(\varphi) q_{M}(\varphi)}{1-q_{M}^{2}(\varphi)}
\end{gathered}
$$

and

$$
\Gamma_{M, 3}(\varphi)=\frac{1}{1-q_{M}^{2}(\varphi)}\left[\Gamma_{M, 1}(\varphi)-q_{M}(\varphi) \Gamma_{M, 2}(\varphi)\right]
$$

with $\beta_{M}(\varphi)$ and $\gamma_{M}(\varphi)$ defined as in (24).

\section{Remarks}

i) For $M=2$ we have $\Gamma_{M, 1}(\varphi)=\Gamma_{M, 2}(\varphi)=0$ and the denominator in (38) vanishes. Such a result confirms that $\varphi$ cannot be estimated when $M<3$.

ii) Using the fourth-order Maclaurin series of $q_{M}(\varphi)$

$$
q_{M}(\varphi) \simeq 1-\frac{M^{2}-1}{6} \varphi^{2}+\frac{\left(M^{2}-1\right)\left(3 M^{2}-7\right)}{360} \varphi^{4}
$$

it is found that, for small values of $\varphi$, functions $\Gamma_{M, i}(\varphi)(i=$ 1,2 ) can be approximated as

$$
\Gamma_{M, i}(\varphi) \simeq \frac{M^{2}-4}{15} \varphi^{2} \quad i=1,2
$$

while $\Gamma_{M, 3}(\varphi) \simeq \Gamma_{M, 1}(\varphi) / 2$. Substituting these results into (38) produces

$\left.\mathrm{E}\left\{\varepsilon_{J M L}^{2}\right\}\right|_{\varphi \rightarrow 0} \simeq \frac{90 \sigma_{w}^{2}}{M\left(M^{2}-1\right)\left(M^{2}-4\right)\|\mathbf{a}+\mathbf{b}\|^{2}}\left(1+\frac{P \sigma_{w}^{2}}{M\|\mathbf{a}+\mathbf{b}\|^{2}}\right)$

which indicates that the accuracy of JML rapidly degrades as $\varphi$ approaches zero. The reason is that the two spectral lines in (12) collapse into a single dc component when $\varphi=0$, thereby preventing the joint estimation of $\mathbf{a}$ and $\mathbf{b}$.

iii) In the absence of any I/Q imbalance we have $\mathbf{b}=\mathbf{0}$ and (38) takes the form

$$
\begin{aligned}
\left.\mathrm{E}\left\{\varepsilon_{J M L}^{2}\right\}\right|_{\mathbf{b}=\mathbf{0}} & =\frac{6 \sigma_{w}^{2}}{M\left(M^{2}-1\right)\|\mathbf{a}\|^{2}} \cdot \frac{1}{\Gamma_{M, 1}(\varphi)} \\
& +\frac{12 P \sigma_{w}^{4}}{M^{2}\left(M^{2}-1\right)\|\mathbf{a}\|^{4}} \cdot \frac{\Gamma_{M, 3}(\varphi)}{\Gamma_{M, 1}^{2}(\varphi)}
\end{aligned}
$$

which, at relatively high SNR values, reduces to

$$
\left.\mathrm{E}\left\{\varepsilon_{J M L}^{2}\right\}\right|_{\mathbf{b}=\mathbf{0},\|\mathbf{a}\|^{2} / \sigma_{w}^{2} \rightarrow \infty}=\frac{6 \sigma_{w}^{2}}{M\left(M^{2}-1\right)\|\mathbf{a}\|^{2}} \cdot \frac{1}{\Gamma_{M, 1}(\varphi)} .
$$

Comparing (29) with (46) and recalling that $0 \leq \Gamma_{M, 1}(\varphi) \leq 1$, it turns out that CML outperforms (at least asymptotically) JML when applied to an ideal receiver with no I/Q imbalance. This result is not surprising since, in the considered scenario, $\hat{\varphi}_{C M L}$ is the ML estimate of $\varphi$.

\section{CONSTRAined JOINT ML ESTIMATION OF THE UNKNOWN PARAMETERS}

\section{A. Estimator's design}

JML is derived without considering the fact that in a practical situation we have $\|\mathbf{a}\| \gg\|\mathbf{b}\|$. We now illustrate how such a side information can be exploited to improve the performance of JML. Our approach aims at maximizing (31) subject to a constraint on the SIR. The resulting scheme is referred to as the constrained JML (CJML) and solves the problem

$$
\begin{array}{ll}
\min _{\tilde{\boldsymbol{\varphi}}, \tilde{\boldsymbol{\theta}}} & \sum_{\substack{p=0 \\
\text { s.t. }}}^{P-1}\left\|\mathbf{x}(p)-\mathbf{A}_{2}(\tilde{\varphi}) \tilde{\boldsymbol{\theta}}(p)\right\|^{2} \\
\text { i } & \|\|^{2} \leq \delta\|\tilde{\mathbf{a}}\|^{2}
\end{array}
$$

where $\delta>0$ is a design parameter. In Appendix B it is shown that CJML takes the form

$$
\hat{\varphi}_{C J M L}=\arg \max _{\tilde{\varphi} \in[-\pi, \pi)}\left\{\Psi_{C J M L}(\tilde{\varphi})\right\}
$$

where the metric $\Psi_{C J M L}(\tilde{\varphi})$ is found to be

$$
\Psi_{C J M L}(\tilde{\varphi})=\sum_{m=0}^{M-1} \sum_{k=0}^{M-1} \chi_{C J M L, m, k}(\tilde{\varphi}) \mathbf{x}_{m}^{H} \mathbf{x}_{k}
$$


with

In such a case it is found that

$$
\begin{aligned}
& \chi_{C J M L, m, k}(\tilde{\varphi})= \\
& \left\{2 \zeta_{1}(\tilde{\varphi})-M\left[\zeta_{1}^{2}(\tilde{\varphi})-2 q_{M}(\tilde{\varphi}) \zeta_{1}(\tilde{\varphi}) \zeta_{2}(\tilde{\varphi})+\zeta_{2}^{2}(\tilde{\varphi})\right]\right\} e^{j(m-k) \varphi^{\delta \rightarrow+\infty}} \chi_{C J M L, m, k}(\tilde{\varphi})= \\
& +2\left\{M\left[\zeta_{1}(\tilde{\varphi})+\zeta_{3}(\tilde{\varphi})\right] \zeta_{2}(\tilde{\varphi})-M q_{M}(\tilde{\varphi})\left[\zeta_{1}(\tilde{\varphi}) \zeta_{3}(\tilde{\varphi})+\zeta_{2}^{2}(\tilde{\varphi})\right]\right. \\
& \left.-2 \zeta_{2}(\tilde{\varphi})\right\} \cos [(m+k-M+1) \tilde{\varphi}] \\
& \zeta_{1}(\tilde{\varphi})=[M+\lambda(\tilde{\varphi})] / D(\tilde{\varphi}) \\
& \zeta_{2}(\tilde{\varphi})=M q_{M}(\tilde{\varphi}) / D(\tilde{\varphi}) \\
& \zeta_{3}(\tilde{\varphi})=[M-\delta \lambda(\tilde{\varphi})] / D(\tilde{\varphi})
\end{aligned}
$$$$
+\left\{2 \zeta_{3}(\tilde{\varphi})-M\left[\zeta_{3}^{2}(\tilde{\varphi})-2 q_{M}(\tilde{\varphi}) \zeta_{2}(\tilde{\varphi}) \zeta_{3}(\tilde{\varphi})+\zeta_{2}^{2}(\tilde{\varphi})\right]\right\} e^{-j(m-k) \tilde{\varphi}}
$$$$
\frac{2}{M} \cdot \frac{\cos [(m-k) \tilde{\varphi}]-q_{M}(\tilde{\varphi}) \cos [(m+k-M+1) \tilde{\varphi}]}{1-q_{M}^{2}(\tilde{\varphi})}
$$

In the above equation, functions $\zeta_{1}(\tilde{\varphi}), \zeta_{2}(\tilde{\varphi})$ and $\zeta_{3}(\tilde{\varphi})$

with $D(\tilde{\varphi})=[M+\lambda(\tilde{\varphi})][M-\delta \lambda(\tilde{\varphi})]-M^{2} q_{M}^{2}(\tilde{\varphi})$ and

$$
\lambda(\tilde{\varphi})=\max \left(0, \frac{\Upsilon_{2}(\tilde{\varphi})-\sqrt{\Upsilon_{2}^{2}(\tilde{\varphi})-\Upsilon_{1}(\tilde{\varphi}) \Upsilon_{3}(\tilde{\varphi})}}{\Upsilon_{1}(\tilde{\varphi})}\right) .
$$

Furthermore, we have

$$
\begin{gathered}
\Upsilon_{1}(\tilde{\varphi})=\delta\left(\delta\left\|\mathbf{t}_{2}(\tilde{\varphi})\right\|^{2}-\left\|\mathbf{t}_{1}(\tilde{\varphi})\right\|^{2}\right) \\
\Upsilon_{2}(\tilde{\varphi})=M \delta\left[\left\|\mathbf{t}_{1}(\tilde{\varphi})\right\|^{2}+\left\|\mathbf{t}_{2}(\tilde{\varphi})\right\|^{2}\right. \\
\left.-2 q_{M}(\tilde{\varphi}) \operatorname{Re}\left\{\mathbf{t}_{1}^{H}(\tilde{\varphi}) \mathbf{t}_{2}(\tilde{\varphi})\right\}\right] \\
\Upsilon_{3}(\tilde{\varphi})=M^{2}\left\{\left[q_{M}^{2}(\tilde{\varphi})-\delta\right]\left\|\mathbf{t}_{1}(\tilde{\varphi})\right\|^{2}\right. \\
\left.-2 q_{M}(\tilde{\varphi})(1-\delta) \operatorname{Re}\left\{\mathbf{t}_{1}^{H}(\tilde{\varphi}) \mathbf{t}_{2}(\tilde{\varphi})\right\}+\left[1-\delta q_{M}^{2}(\tilde{\varphi})\right]\left\|\mathbf{t}_{2}(\tilde{\varphi})\right\|^{2}\right\}
\end{gathered}
$$

where $\mathbf{t}_{1}$ and $\mathbf{t}_{2}$ are $P$-dimensional vectors with entries $\left[\mathbf{t}_{1}(\tilde{\varphi})\right]_{p}=\mathbf{u}^{H}(\tilde{\varphi}) \mathbf{x}(p)$ and $\left[\mathbf{t}_{2}(\tilde{\varphi})\right]_{p}=\mathbf{u}^{H}(-\tilde{\varphi}) \mathbf{x}(p)$ for $p=0,1, \ldots, P-1$.

Since evaluating the theoretical performance of CJML is extremely challenging, the accuracy of this scheme will be assessed in Sect. VII by means of numerical simulations.

\section{B. Remarks}

i) When $\delta$ approaches zero, we have $\lim _{\delta \rightarrow 0} \lambda(\tilde{\varphi})=+\infty$ and $\lim _{\delta \rightarrow 0} \delta \lambda(\tilde{\varphi})=0$. Hence, from (51)-(53) it is found that $\zeta_{1}(\tilde{\varphi})$ approaches $1 / M$, while $\zeta_{2}(\tilde{\varphi})$ and $\zeta_{3}(\tilde{\varphi})$ become vanishingly small. This leads to

$$
\lim _{\delta \rightarrow 0} \chi_{C J M L, m, k}(\tilde{\varphi})=\frac{1}{M} e^{j(m-k) \tilde{\varphi}}=\frac{1}{M} \chi_{C M L, m, k}(\tilde{\varphi})
$$

which means that CJML reduces to CML. The reason is that letting $\delta=0$ in the constraint $\|\mathbf{b}\|^{2} \leq \delta\|\mathbf{a}\|^{2}$ amounts to putting $\mathbf{b}=\mathbf{0}$, which is just the underlying assumption of CML.

ii) When $\delta$ goes to infinity, we have $\lim _{\delta \rightarrow+\infty} \lambda(\tilde{\varphi})=$ $\lim _{\delta \rightarrow+\infty} \delta \lambda(\tilde{\varphi})=0$, leading to

$$
\begin{gathered}
\lim _{\delta \rightarrow+\infty} \zeta_{1}(\tilde{\varphi})=\lim _{\delta \rightarrow+\infty} \zeta_{3}(\tilde{\varphi})=\frac{1}{M\left[1-q_{M}^{2}(\tilde{\varphi})\right]} \\
\lim _{\delta \rightarrow+\infty} \zeta_{2}(\tilde{\varphi})=\frac{q_{M}(\tilde{\varphi})}{M\left[1-q_{M}^{2}(\tilde{\varphi})\right]} .
\end{gathered}
$$

which, compared with (37), reveals that CJML reduces to JML. This fact can be explained by observing that letting $\delta \rightarrow+\infty$ amounts to removing any constraint on the magnitude of $\mathbf{b}$.

The above remarks qualify CJML as a general ML-based estimator, which incorporates both CML and JML as special cases when $\delta \rightarrow 0$ and $\delta \rightarrow+\infty$, respectively.

\section{Computational COMPLEXity of CML, JML AND CJML}

\section{A. CML Algorithm}

In this section we assess the complexity of the investigated schemes in terms of real multiplications (RMs) and real additions (RAs). For this purpose, we observe that a complex multiplication is equivalent to four RMs plus two RAs, while a complex addition involves two RAs.

We start by rewriting (17) in the form

$$
\Psi_{C M L}(\tilde{\varphi})=\left\|\mathbf{t}_{1}(\tilde{\varphi})\right\|^{2}
$$

where $\left[\mathbf{t}_{1}(\tilde{\varphi})\right]_{p}=\mathbf{u}^{H}(\tilde{\varphi}) \mathbf{x}(p)$, for $p=0,1, \ldots, P-1$. Since the computation of $\left[\mathbf{t}_{1}(\tilde{\varphi})\right]_{p}$ requires $M$ complex multiplications and $M-1$ complex additions, evaluating $\mathbf{t}_{1}(\tilde{\varphi})$ needs $4 P M$ RMs and $4 P M-2 P$ RAs. Additional $2 P$ RMs and $2 P-1$ RAs are required to obtain $\left\|\mathbf{t}_{1}(\tilde{\varphi})\right\|^{2}$, so that computing $\Psi_{C M L}(\tilde{\varphi})$ for each $\tilde{\varphi}$ needs $4 P M+2 P$ RMs and $4 P M-1$ RAs.

\section{B. JML Algorithm}

The complexity of JML is assessed by reformulating (35) as

$$
\begin{array}{r}
\Psi_{J M L}(\tilde{\varphi})=\frac{1}{1-q_{M}^{2}(\tilde{\varphi})}\left[\left\|\mathbf{t}_{1}(\tilde{\varphi})\right\|^{2}+\left\|\mathbf{t}_{2}(\tilde{\varphi})\right\|^{2}\right. \\
\left.-2 q_{M}(\tilde{\varphi}) \operatorname{Re}\left\{\mathbf{t}_{1}^{H}(\tilde{\varphi}) \mathbf{t}_{2}(\tilde{\varphi})\right\}\right]
\end{array}
$$

where $\left[\mathbf{t}_{2}(\tilde{\varphi})\right]_{p}=\mathbf{u}^{H}(-\tilde{\varphi}) \mathbf{x}(p)$ for $p=0,1, \ldots, P-1$. Based on the results obtained for the CML algorithm, it is shown that the computation of a single value of $\Psi_{J M L}(\tilde{\varphi})$ requires $8 P M+6 P+4$ RMs plus $8 P M+2 P$ RAs.

\section{CJML Algorithm}

We first observe that, once $\mathbf{t}_{1}(\tilde{\varphi})$ and $\mathbf{t}_{2}(\tilde{\varphi})$ have been computed, evaluating $\Upsilon_{1}(\tilde{\varphi}), \Upsilon_{2}(\tilde{\varphi})$, and $\Upsilon_{3}(\tilde{\varphi})$ through (55)-(57) requires additional $6 P+14 \mathrm{RMs}$ and $6 P+5 \mathrm{RAs}$. Also, given $\Upsilon_{1}(\tilde{\varphi}), \Upsilon_{2}(\tilde{\varphi})$, and $\Upsilon_{3}(\tilde{\varphi})$, the computation of $\lambda(\tilde{\varphi})$ through (54) involves 4 RMs and 2 RAs. Considering the calculation of $\mathbf{t}_{1}(\tilde{\varphi})$ and $\mathbf{t}_{2}(\tilde{\varphi})$, we conclude that computing $\lambda(\tilde{\varphi})$ requires a total of $8 P M+6 P+18 \mathrm{RMs}$ and $8 P M+2 P+7$ RAs. 
TABLE I

COMPLEXITY OF THE INVESTIGATED SCHEMES.

\begin{tabular}{||c|c|c||}
\hline Algorithm & Real operations & WLAN scenario \\
\hline \hline CML & $8 P M+2 P-1$ & 544 \\
\hline JML & $16 P M+8 P+4$ & 1124 \\
\hline CJML & $16 P M+48 P+28$ & 1510 \\
\hline
\end{tabular}

Now, we focus on the computation of $\Psi_{C J M L}(\tilde{\varphi})$ through (85) which, after neglecting irrelevant terms independent of $\tilde{\varphi}$, is equivalent to

$$
\begin{array}{r}
\Psi_{C J M L}(\tilde{\varphi})=M\|\hat{\mathbf{a}}\|^{2}+M\|\hat{\mathbf{b}}\|^{2}-2 \operatorname{Re}\left\{\hat{\mathbf{a}}^{H} \mathbf{t}_{1}(\tilde{\varphi})\right\} \\
-2 \operatorname{Re}\left\{\hat{\mathbf{b}}^{H} \mathbf{t}_{2}(\tilde{\varphi})\right\}+2 M q_{M}(\varphi) \operatorname{Re}\left\{\hat{\mathbf{b}}^{H} \hat{\mathbf{a}}\right\}
\end{array}
$$

Assuming that $\lambda(\tilde{\varphi})$, and hence $\mathbf{u}^{H}(\tilde{\varphi}) \mathbf{x}(p)=\left[\mathbf{t}_{1}(\tilde{\varphi})\right]_{p}$ and $\mathbf{u}^{H}(-\tilde{\varphi}) \mathbf{x}(p)=\left[\mathbf{t}_{2}(\tilde{\varphi})\right]_{p}$, are available, the calculation of $\hat{\mathbf{a}}$ and $\hat{\mathbf{b}}$ through $(84 \mathrm{a})-(84 \mathrm{~b})$ requires a total of $13 P$ RMs and $7 P$ RAs. Additional $2 P$ RMs and $2 P-1$ RAs are required for the computation of each quantity $\|\hat{\mathbf{a}}\|^{2},\|\hat{\mathbf{b}}\|^{2}, \operatorname{Re}\left\{\hat{\mathbf{a}}^{H} \mathbf{t}_{1}(\tilde{\varphi})\right\}$, $\operatorname{Re}\left\{\hat{\mathbf{b}}^{H} \mathbf{t}_{2}(\tilde{\varphi})\right\}$ and $\operatorname{Re}\left\{\hat{\mathbf{b}}^{H} \hat{\mathbf{a}}\right\}$, while 4 additional RMs and 4 RAs are needed for evaluating the right-hand side of (62). It can be concluded that the computation of $\Psi_{C J M L}(\tilde{\varphi})$ for each $\tilde{\varphi}$ requires a total of $8 P M+29 P+22 \mathrm{RMs}$ and $8 P M+19 P+6$ RAs.

Table I summarizes the number of real operations involved in the computation of $\Psi_{C M L}(\tilde{\varphi}), \Psi_{J M L}(\tilde{\varphi})$, and $\Psi_{C J M L}(\tilde{\varphi})$ as a function of $M$ and $P$. The rightmost column reports the overall complexity required in a WLAN scenario, where the useful part of the TP (excluding the CP) is composed by $M=$ 8 repeated segments carrying $P=16$ samples. These figures indicate that CJML is computationally more demanding than CML and JML, since it leads to an increase of the system complexity by a factor 2.8 and 1.3 , respectively.

\section{CRB ANALYSIS}

It is interesting to compare the performance of the estimation algorithms illustrated in the previous section with the relevant CRB. The latter is computed from (30) using the true statistical distribution of $w_{I}(t)$ and $w_{Q}(t)$ as given in (4). For this purpose, we arrange the samples $x_{m}(p)=x_{m}^{I}(p)+j x_{m}^{Q}(p)$ into a real-valued vector $\mathbf{x}=\left[x_{0}^{I}(0), x_{0}^{Q}(0), x_{0}^{I}(1), x_{0}^{Q}(1) \cdots\right.$ $\left.x_{M-1}^{I}(P-1), x_{M-1}^{Q}(P-1)\right]^{T}$ with $2 P M$ entries. Then, from (6) we can write

$$
\mathbf{x}=\boldsymbol{\eta}+\mathbf{w}
$$

where $\mathbf{w}=\left[w_{0}^{I}(0), w_{0}^{Q}(0), w_{0}^{I}(1), w_{0}^{Q}(1) \cdots w_{M-1}^{I}(P-\right.$ 1), $\left.w_{M-1}^{Q}(P-1)\right]^{T}$ is the noise contribution, with $w_{m}^{I}(p)$ and $w_{m}^{Q}(p)$ being the real and imaginary parts of $w_{m}(p)$, respectively. Furthermore, letting $a(p)=a^{I}(p)+j a^{Q}(p)$ and $b(p)=b^{I}(p)+j b^{Q}(p)$, we have

$$
\eta=\mathbf{Q z}
$$

with $\mathbf{z}=\left[\begin{array}{llll}\mathbf{z}^{T}(0) & \mathbf{z}^{T}(1) & \cdots & \mathbf{z}^{T}(P-1)\end{array}\right]^{T}$ and $\mathbf{z}(p)=$ $\left[a^{I}(p), a^{Q}(p), b^{I}(p), b^{Q}(p)\right]^{T}$, while $\mathbf{Q}$ is a matrix of dimension $2 P M \times 4 P$ with the following structure

$$
\mathbf{Q}=\left[\begin{array}{llll}
\mathbf{Q}_{0}^{T} & \mathbf{Q}_{1}^{T} & \cdots & \mathbf{Q}_{M-1}^{T}
\end{array}\right]^{T} .
$$

In the above equation, $\mathbf{Q}_{m}$ is a $2 P \times 4 P$ matrix

$$
\mathbf{Q}_{m}=\operatorname{diag}\{\underbrace{\mathbf{R}_{m}, \mathbf{R}_{m}, \ldots, \mathbf{R}_{m}}_{P}\} \quad m=0,1, \ldots, M-1
$$

where $\mathbf{R}_{m}$ is defined as

$$
\mathbf{R}_{m}=\left[\begin{array}{cccc}
\mathrm{c}_{m}(\varphi) & -\mathrm{s}_{m}(\varphi) & \mathrm{c}_{m}(\varphi) & \mathrm{s}_{m}(\varphi) \\
\mathrm{s}_{m}(\varphi) & \mathrm{c}_{m}(\varphi) & -\mathrm{s}_{m}(\varphi) & \mathrm{c}_{m}(\varphi)
\end{array}\right]
$$

with $\mathrm{c}_{m}(\varphi)$ and $\mathrm{s}_{m}(\varphi)$ being a shorthand notation for $\cos \left[\left(m-\frac{M-1}{2}\right) \varphi\right]$ and $\sin \left[\left(m-\frac{M-1}{2}\right) \varphi\right]$, respectively. For notational simplicity, in (65) we have omitted the dependence of $\mathbf{Q}$ on $\varphi$.

In Appendix $\mathrm{C}$ it is shown that

$$
\operatorname{CRB}(\varphi)=\frac{1}{\mathbf{z}^{T} \dot{\mathbf{Q}}^{T}\left[\mathbf{C}_{w}^{-1}-\mathbf{C}_{w}^{-1} \mathbf{Q}\left(\mathbf{Q}^{T} \mathbf{C}_{w}^{-1} \mathbf{Q}\right)^{-1} \mathbf{Q}^{T} \mathbf{C}_{w}^{-1}\right] \dot{\mathbf{Q}} \mathbf{z}}
$$

where $\mathbf{C}_{w}$ is the correlation matrix of $\mathbf{w}$ and $\dot{\mathbf{Q}}$ is the derivative of $\mathbf{Q}$ with respect to $\varphi$. A simpler expression is obtained by assuming a white-noise scenario wherein $\mathbf{C}_{w}=$ $\left(\sigma_{w}^{2} / 2\right) \mathbf{I}_{2 P M}$. In such a case, after lengthy computations it is found that (68) takes the form

$$
\operatorname{CRB}(\varphi)=\frac{6 \sigma_{w}^{2}\left[M\left(M^{2}-1\right)\right]^{-1}}{\left[\Gamma_{M, 1}(\varphi)\left(\|\mathbf{a}\|^{2}+\|\mathbf{b}\|^{2}\right)+2 \Gamma_{M, 2}(\varphi) \operatorname{Re}\left(\mathbf{a}^{H} \mathbf{b}\right)\right]}
$$

with $\Gamma_{M, 1}(\varphi)$ and $\Gamma_{M, 2}(\varphi)$ defined as in (39) and (40). It is worth noting that, at relatively high SNR values, the accuracy of $\hat{\varphi}_{J M L}$ given in (38) approaches the CRB in (69), meaning that JML is asymptotically efficient in the presence of AWGN.

\section{Simulation RESUlTS}

\section{A. Simulation model}

The investigated system is compliant with the IEEE 802.11a standard for WLANs [17]. Specifically, the DFT size is $N=$ 64 with a signaling interval $T_{s}=50 \mathrm{~ns}$ which corresponds to a subcarrier distance of $312.5 \mathrm{kHz}$. The TP is composed by ten repeated segments of length $P=16$. By considering the first two segments as the $\mathrm{CP}$ of the $\mathrm{TP}$, the remaining $M=8$ segments are exploited for CFO recovery. We adopt a discretetime channel model and collect the $T_{s}$-spaced samples of $v(t)$ into a vector $\mathbf{v}=\left[v(0), v(1), \ldots, v\left(L_{v}-1\right)\right]^{T}$. The entries of $\mathbf{v}$ are independent and circularly symmetric Gaussian random variables with zero-mean and power

$$
\mathrm{E}\left\{|v(k)|^{2}\right\}=\sigma_{v}^{2} \exp \left(-k / L_{v}\right) \quad k=0,1, \ldots, L_{v}-1
$$

where $\sigma_{v}^{2}$ is chosen such that $\mathrm{E}\left\{\|\mathbf{v}\|^{2}\right\}=1$. Unless otherwise specified, we consider the following two scenarios [7]:

1) Frequency-selective $I / Q$ imbalance $(F S-I / Q)$ : the analog I/Q filters have discrete-time impulse responses $\mathbf{g}_{I}=[0,1, \mu]^{T}$ and $\mathbf{g}_{Q}=[\mu, 1,0]^{T}$ with $\mu=0.1$, while the LO-induced imbalance is characterized by $\alpha=1.122(1 \mathrm{~dB})$ and $\psi=5$ degrees. From (2), it follows that $h(k)$ and $q(k)$ have support $k=0,1, \ldots, L-1$, with $L=L_{v}+2$.

2) Frequency-flat $I / Q$ imbalance $(F F-I / Q)$ : only frequency independent imbalance is considered with $\alpha=1.122$ and $\psi=$ $5^{\mathrm{o}}$, while the I/Q filters have ideal response $[0,1,0]^{T}$. 


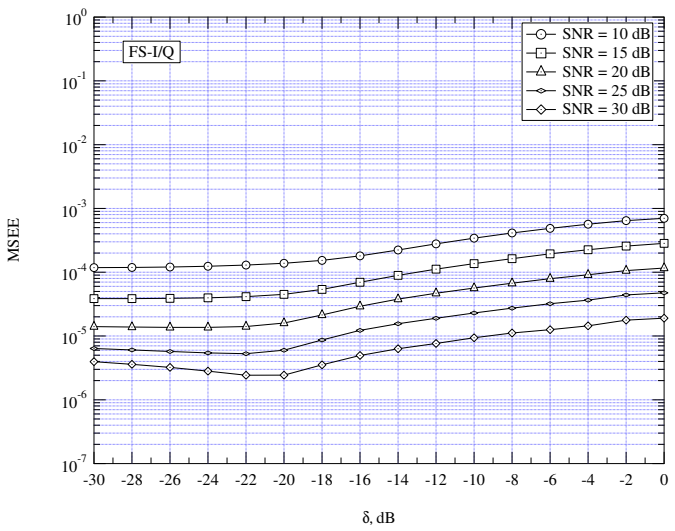

Fig. 2. Accuracy of CJML vs $\delta$ for different SNR values in the FS-I/Q scenario.

In order to assess the sensitivity of the considered schemes to the amount of RF imperfections, we also consider a general set-up wherein a coefficient $\rho \in[0,4]$ is used to specify the I/Q imbalance parameters as $\mu=0.1 \rho, \alpha=1+0.122 \rho$ and $\psi=5 \rho$ degrees. Clearly, $\rho=0$ corresponds to the absence of any I/Q imbalance, while $\rho=1$ yields the FS-I/Q scenario.

The average SIR is defined in [7] and can expressed as

$$
\operatorname{SIR}=\frac{\left(1+\alpha^{2}\right)\left(1+\mu^{2}\right)+2 \alpha \cos \psi}{\left(1+\alpha^{2}\right)\left(1+\mu^{2}\right)-2 \alpha \cos \psi}
$$

yielding the values of $19.9 \mathrm{~dB}$ and $22.8 \mathrm{~dB}$ for the FS-I/Q and FF-I/Q cases, respectively.

Assuming a carrier frequency of $5 \mathrm{GHz}$ and an oscillator instability of \pm 30 parts-per-million (ppm), the maximum value of the normalized CFO is approximately given by $\nu_{\max }=0.5$. Hence, recalling that $Q=N / P=4$, from (7) it follows that $\varphi \in[-\pi / 4, \pi / 4]$. The global maximum of the CFO metrics shown in (18), (36) and (49) is found by evaluating the metric over a grid of $K$ uniformly-spaced values $\tilde{\varphi}_{k}=-\pi / 4+k \pi /(2 K)$ for $k=0,1, \ldots, K$ (coarse search), followed by a parabolic interpolation (fine search). Parameter $K$ has been set to 128 since no significant improvement is achieved when using $K>128$.

\section{B. Performance assessment for FO estimation}

An important design parameter for CJML is the coefficient $\delta$, which specifies the constraint on the SIR level. Fig. 2 shows the accuracy of CJML as a function of $\delta$ for different SNR values and with $\varphi$ uniformly distributed over the range $[-\pi / 4, \pi / 4]$. These results are obtained in the FS-I/Q scenario, and are qualitatively similar to those pertaining to the FF$\mathrm{I} / \mathrm{Q}$ case (not shown for space limitations). As is seen, at intermediate and low SNR values the MSEE monotonically increases with $\delta$, while at high SNR values a global minimum occurs around $\delta=-22 \mathrm{~dB}$. Extensive numerical measurements carried out in the general set-up with $\rho \in[0,4]$ indicate that nearly optimal performance can be achieved by letting $\delta=(\mathrm{SIR})^{-1}$, which is therefore used in all subsequent simulations.
Figs. 3 and 4 illustrate the MSEE of the CFO estimators as a function of $\rho$ with $\varphi$ uniformly distributed over $[-\pi / 4, \pi / 4]$. The SNR is $15 \mathrm{~dB}$ in Fig. 3 and $30 \mathrm{~dB}$ in Fig. 4. The solid line illustrates theoretical analysis for CML, while for JML and CJML it is used to facilitate the reading of the plot. It turns out that the accuracy of JML is virtually independent of $\rho$, while CML exhibits a remarkable sensitivity to the amount of I/Q imbalances. However, at $\mathrm{SNR}=15 \mathrm{~dB}$ the $\mathrm{CML}$ outperforms JML for all the considered values of $\rho$, while at $\mathrm{SNR}=30 \mathrm{~dB}$ CML is worse than JML only for $\rho>1.9$. These results indicate that, contrary to the well-established belief, CML performs satisfactorily in most practical situations and the adoption of more sophisticated schemes is justified only at high SNR values and in the presence of extremely severe $\mathrm{RF}$ imbalances. We also see that, in the presence of nonnegligible I/Q imbalances, the best accuracy is achieved by CJML. The reason is that this scheme is able to find a good balance between CML and JML thanks to a proper design of $\delta$. In particular, for $\rho=0$ we have $\delta=0$ and CJML reduces to CML, while for large values of $\rho$ it departs from CML and approaches JML.

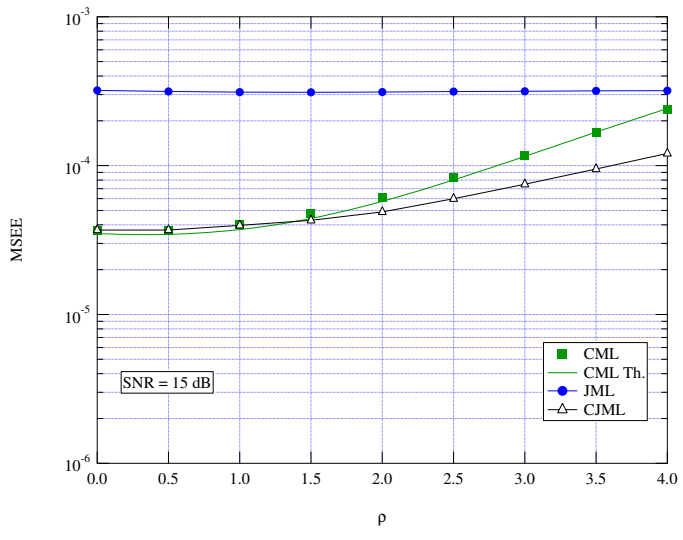

Fig. 3. Accuracy of the CFO estimators vs $\rho$ with $\mathrm{SNR}=15 \mathrm{~dB}$.

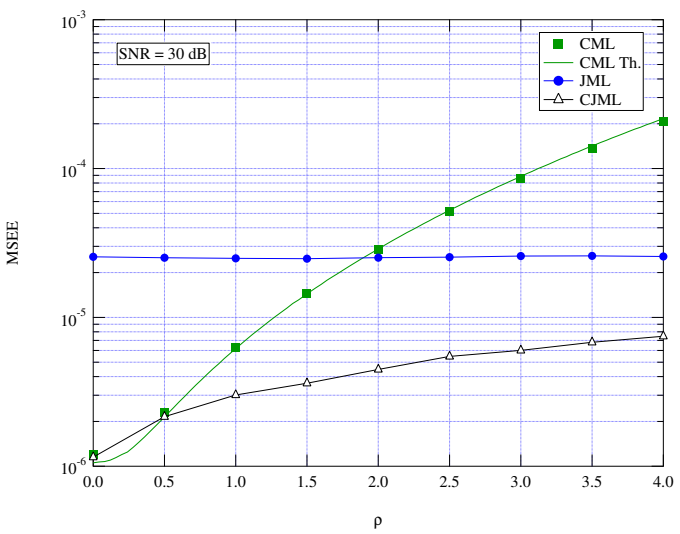

Fig. 4. Accuracy of the CFO estimators vs $\rho$ with $\mathrm{SNR}=30 \mathrm{~dB}$.

Fig. 5 illustrates the MSEE of the CFO estimators as a function of $\varphi$ measured at SNR $=15 \mathrm{~dB}$ in the FS$\mathrm{I} / \mathrm{Q}$ scenario. The CRB reported in (69) is also shown for 
comparison. As expected, JML performs poorly for small CFO

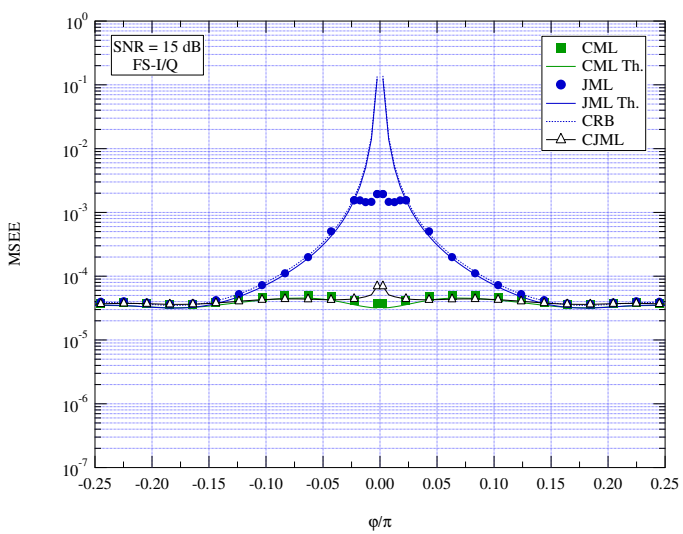

Fig. 5. Accuracy of the CFO estimators vs $\varphi$ in the FS-I/Q scenario with $\mathrm{SNR}=15 \mathrm{~dB}$.

values since in this case the useful signal component and its mirror image collapse into a single dc line and cannot be easily resolved. This is also reflected in the CRB curve, which goes to infinity as $\varphi$ approaches zero. In contrast, the accuracy of both CML and JCML depends weakly on the CFO value and is remarkably better than that of JML for $|\varphi|<0.1 \pi$. Since CML is derived by ignoring the presence of I/Q imbalances, the fact that this scheme outperforms JML may appear surprising. Actually, such a behaviour can be explained by observing that for $\varphi=0$ the received signal in (12) reduces to a dc line embedded in (approximately) white Gaussian noise and, due to the absence of any mirror interference, CML provides nearly optimum performance. On the other hand, in this scenario JML cannot work properly due to the impossibility of providing independent estimates of the nuisance vectors $\mathbf{a}$ and $\mathbf{b}$. It is worth noting that the theoretical analysis of CML and JML is in good agreement with simulation results except when we consider JML at small CFO values. Such a discrepancy is due to the fact that the MSEE shown in (38) is derived using the approach of [22], which is valid in the presence of small estimation errors. It is also worth recalling that no tangible difference has been observed between the true CRB (68) and its approximation (69), meaning that the noise term $w(t)$ in (3) can reasonably be approximated as a circularly symmetric wihite Gaussian process.

The results shown in Fig. 6 are obtained under the same operating conditions of Fig. 5, except that the SNR is now set to $30 \mathrm{~dB}$. In this case, we see that CML outperforms JML only when $|\varphi|$ is approximately smaller than $0.05 \pi$. Such behaviour is justified by the fact that, at large SNR values, the MSEE of JML becomes proportional to $(\mathrm{SNR})^{-1}$, while the accuracy of CML is essentially determined by the bias term $\mathrm{E}^{2}\left\{\varepsilon_{C M L}\right\}$ present in (25), which vanishes only for specific values of $\varphi$. The CJML provides better estimates than CML except in the proximity of $\varphi=0$. Compared to JML, it performs slightly worse when $|\varphi|>0.05 \pi$, while a significant improvement is observed at smaller CFO values.

Fig. 7 illustrates the bias of the investigates schemes as a function of $\varphi$ in the FS-I/Q scenario with the SNR fixed to

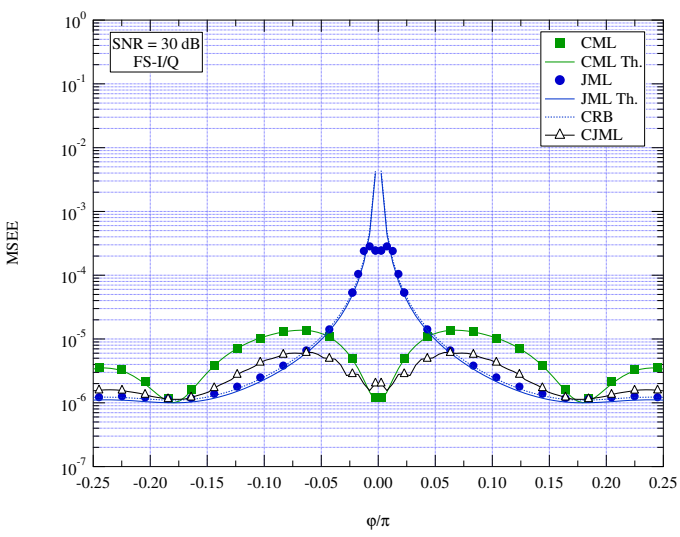

Fig. 6. Accuracy of the CFO estimators vs $\varphi$ in the FS-I/Q scenario with $\mathrm{SNR}=30 \mathrm{~dB}$

$30 \mathrm{~dB}$. As is seen, the bias of CJML and CML is smaller than $1.5 \times 10^{-3}$, while higher values are observed with JML. This contradicts the theoretical analysis of Sect. IV.B, where it was shown that $\mathrm{E}\left\{\hat{\varphi}_{J M L}\right\}=\varphi$. Such a discrepancy can be justified by recalling that our theoretical results are accurate only in the presence of small estimation errors.

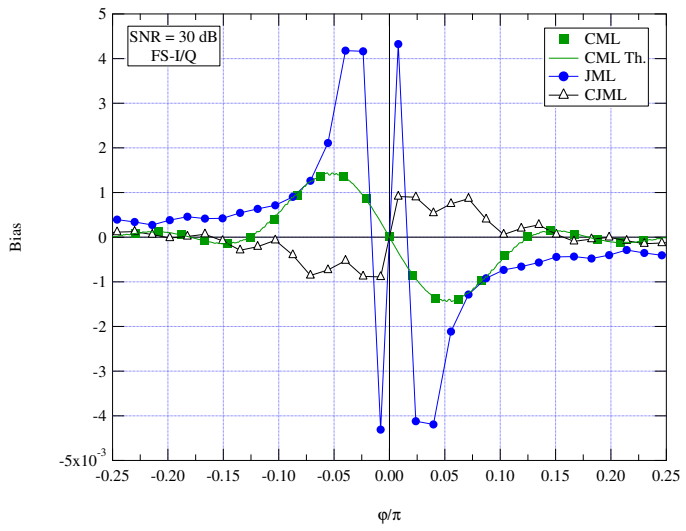

Fig. 7. Bias of the CFO estimates $\varphi$ in the FS-I/Q scenario with $\mathrm{SNR}=30$ dB.

Figs. 8 and 9 illustrate the MSEE of the investigated schemes as a function of the SNR for the FS-I/Q and FF-I/Q scenarios, respectively, when $\varphi$ varies uniformly over the range $[-\pi / 4, \pi / 4]$. Comparisons are made with available CFO recovery methods which exploit a repeated $\mathrm{TP}$ to cope with I/Q imbalances. Specifically, we consider the ESPRIT-based estimator illustrated in [11] and other heuristic algorithms proposed by Pan and Phoong (PP) in [8], by Kume, Lin and Yamashita (KLY) in [10], and by Wang, Xue, Liu, Ye and Ren (WXLYR) in [9]. At SNR values smaller than $24 \mathrm{~dB}$, both CML and CJML outperform all the other methods, with CJML taking the lead as the SNR increases. Compared to CML and CJML, the ESPRIT-based scheme entails a loss of approximately $5 \mathrm{~dB}$ at medium SNR values, which increases to $10 \mathrm{~dB}$ when considering the JML. Such a remarkable loss is due to the poor accuracy of JML in case of small CFOs. The PP algorithm operates satisfactorily at medium-to-high 


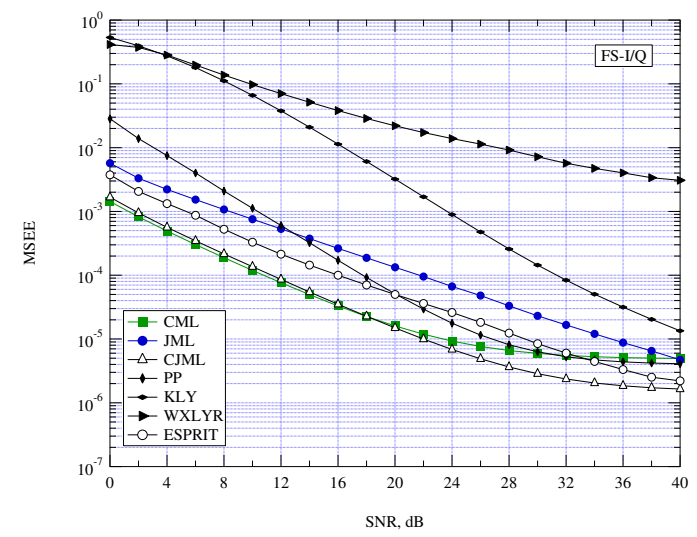

Fig. 8. Accuracy of the CFO estimators vs SNR in the FS-I/Q scenario.

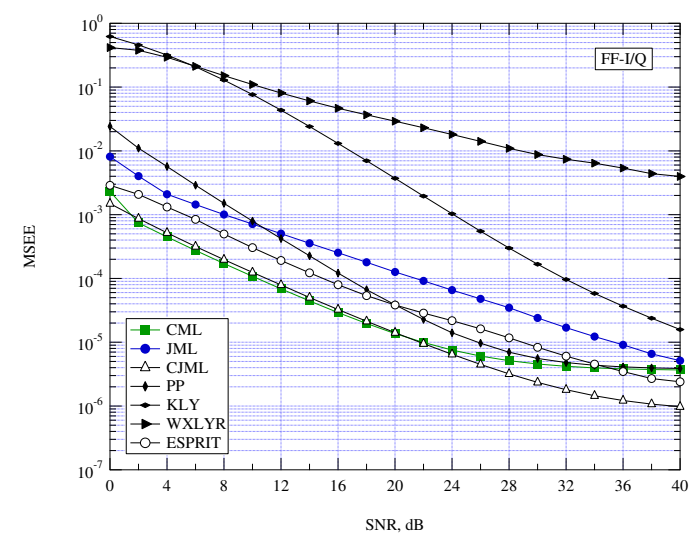

Fig. 9. Accuracy of the CFO estimators vs SNR in the FF-I/Q scenario.

SNR values, while a significant degradation is observed when the SNR decreases. As for KLY and WXLYR, they perform quite poorly. This is particularly evident for the latter scheme, whose MSEE curve is plagued by a considerable floor.

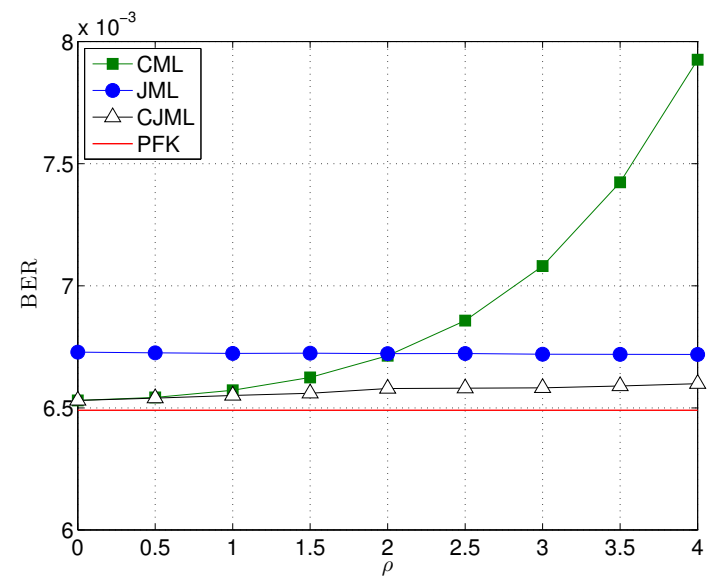

Fig. 10. BER for a 64-QAM modulation vs $\rho$ with $\mathrm{SNR}=30 \mathrm{~dB}$.

Fig. 10 provides the bit-error-rate (BER) performance of an uncoded 64-QAM transmission when CFO correction is accomplished by resorting to CML, JML or CJML. We consider the general simulation set-up with $\rho$ varying in the interval $[0,4]$ and with the SNR value fixed to $30 \mathrm{~dB}$. In order to distinguish the impact of the frequency estimates from that of other system impairments, ideal compensation of the $\mathrm{I} / \mathrm{Q}$ imbalance parameters and ideal channel equalization is assumed. The BER value obtained in the presence of perfect frequency knowledge (PFK) is also shown as a benchmark. As expected, the BER curves exhibit the same trend of the MSEE curves shown in Fig. 4. In particular, we see that the error-rate increases with $\rho$ when using CML, while a reduced sensitivity to the I/Q imbalance is observed when adopting JML and CJML. For $\rho=1$ all the considered schemes provides similar BER results, thereby confirming that CML can perform satisfactorily in most practical situations.

\section{Conclusions}

We have presented an analytical investigation of the frequency recovery problem in a direct-conversion receiver affected by frequency selective I/Q imbalance. The first objective was to check whether traditional CFO estimators can be applied or not to a DCR architecture. For this purpose, we have analytically assessed the impact of the I/Q imbalance on the performance of the conventional ML (CML) scheme. Next, we have reviewed and analyzed the JML method, which provides joint estimates of the CFO, the useful signal component and its mirror image. Finally, we have derived a novel scheme (CJML), which exploits some side-information about the signal-to-interference ratio. It was shown that both CML and JML can be obtained from CJML by properly adjusting the value of a design parameter. In response to the questions raised in Sect. I, the main conclusions that can be drawn from this study are as follows:

1) CML performs satisfactorily in most situations and outperforms JML at SNR values of practical interest in both the FS-I/Q and FF-I/Q scenarios. This result contradicts the common idea that conventional frequency recovery schemes for OFDM systems perform poorly in the presence of I/Q imbalance;

2) CJML is able to get an effective balance between CML and JML, and exhibits an excellent accuracy over a large range of CFO and SNR values at the price of an increased complexity. In a forward-looking perspective, its improved resilience against I/Q imbalances can be exploited to relax the requirements on hardware components for DCR architectures;

3) JML performs poorly for small CFO values and, in the medium SNR range, the MSEE analysis exhibits a loss of approximately $10 \mathrm{~dB}$ with respect to CML and CJML. A remarkable loss is also observed with alternative schemes based on the ESPRIT algorithm or other heuristic methods;

4) The question of whether the improved accuracy of CJML justifies or not its increased complexity with respect to CML is controversial. The answer depends on many different factors, such as the cost of hardware components, the impact of the increased power consumption on the battery life and the relative weight of the CJML 
complexity with respect to that of other fundamental functions, including data decoding. Overall, we expect that such a relative weight is marginal since data decoding must be continuously performed in the receiver, while frequency synchronization is typically accomplished once per frame.

\section{APPENDIX A}

In this Appendix we evaluate the mean and the MSEE of the CML estimate given in (16) under the simplifying assumption that the noise term $w(t)$ in (1) is a ZMCSG complex random process. We begin by taking the derivatives of $\Psi_{C M L}(\varphi)$ in (18), yielding

$$
\begin{aligned}
& \Psi_{C M L}^{\prime}(\varphi)=\sum_{m=0}^{M-1} \sum_{k=0}^{M-1}(k-m) \operatorname{Im}\left\{\mathbf{x}_{m}^{H} \mathbf{x}_{k} e^{j(m-k) \varphi}\right\} \\
& \Psi_{C M L}^{\prime \prime}(\varphi)=-\sum_{m=0}^{M-1} \sum_{k=0}^{M-1}(k-m)^{2} \operatorname{Re}\left\{\mathbf{x}_{m}^{H} \mathbf{x}_{k} e^{j(m-k) \varphi}\right\}
\end{aligned}
$$

and rewrite (6) in vector form as

$$
\mathbf{x}_{m}=\boldsymbol{\eta}_{m}+\mathbf{w}_{m}
$$

where $\boldsymbol{\eta}_{m}=\mathbf{a} e^{j[m-(M-1) / 2] \varphi}+\mathbf{b} e^{-j[m-(M-1) / 2] \varphi}$, while $\left\{\mathbf{w}_{m} ; m=0,1, \ldots, M-1\right\}$ are statistically independent ZMCSG random vectors with covariance matrix $\sigma_{w}^{2} \mathbf{I}_{P}$. Denoting by $\delta(n)$ the Kronecker delta function, from (74) we get

$\mathrm{E}\left\{\mathbf{x}_{m}^{H} \mathbf{x}_{k} e^{j(m-k) \varphi}\right\}=\boldsymbol{\eta}_{m}^{H} \boldsymbol{\eta}_{k} e^{j(m-k) \varphi}+\sigma_{w}^{2} P \delta(m-k) e^{j(m-k) \varphi}$

which, after substituting into (72) and (73), produces

$$
\begin{aligned}
\mathrm{E}\left\{\Psi_{C M L}^{\prime}(\varphi)\right\} & =M^{2} q_{M}^{\prime}(\varphi)\left[q_{M}(\varphi)\|\mathbf{b}\|^{2}+\operatorname{Re}\left(\mathbf{a}^{H} \mathbf{b}\right)\right] \\
\mathrm{E}\left\{\Psi_{C M L}^{\prime \prime}(\varphi)\right\} & =\frac{M^{2}\left(M^{2}-1\right)}{6}\left\{\left[\beta_{M}(\varphi)+q_{M}(\varphi) \gamma_{M}(\varphi)\right]\|\mathbf{b}\|^{2}\right. \\
& \left.-\|\mathbf{a}\|^{2}-\left[q_{M}(\varphi)-\gamma_{M}(\varphi)\right] \operatorname{Re}\left(\mathbf{a}^{H} \mathbf{b}\right)\right\}
\end{aligned}
$$

where $q_{M}(\varphi), \beta_{M}(\varphi)$ and $\gamma_{M}(\varphi)$ are defined in (22) and (24). Finally, inserting these results into (19), yields $\mathrm{E}\left\{\varepsilon_{C M L}\right\}$ as given in (21).

Now, we concentrate on the computation of the MSEE. From (20), it turns out that we need the expectation of $\left[\Psi_{C M L}^{\prime}(\varphi)\right]^{2}$ which, using (72), can be rewritten as

$$
\begin{array}{r}
{\left[\Psi_{C M L}^{\prime}(\varphi)\right]^{2}=-\sum_{m=0}^{M-1} \sum_{k=0}^{M-1} \sum_{n=0}^{M-1} \sum_{\ell=0}^{M-1}(m-k)(n-\ell) \times} \\
\times e^{j(m-k) \varphi} e^{j(n-\ell) \varphi} \mathbf{x}_{m}^{H} \mathbf{x}_{k} \mathbf{x}_{n}^{H} \mathbf{x}_{\ell} .
\end{array}
$$

The expectation of (78) is computed by exploiting the identity $\mathrm{E}\left\{\mathbf{w}_{m}^{H} \mathbf{w}_{k} \mathbf{w}_{n}^{H} \mathbf{w}_{\ell}\right\}=P^{2} \sigma_{w}^{4} \delta(m-k) \delta(n-\ell)+P \sigma_{w}^{4} \delta(m-\ell) \delta(k-$

and is found to be

$$
\begin{aligned}
& \mathrm{E}\left\{\left[\Psi_{C M L}^{\prime}(\varphi)\right]^{2}\right\}=\left[\mathrm{E}\left\{\Psi_{C M L}^{\prime}(\varphi)\right\}\right]^{2}+ \\
& \frac{M^{3}\left(M^{2}-1\right)}{6} A_{M}(\varphi) \sigma_{w}^{2}+P \frac{M^{2}\left(M^{2}-1\right)}{6} \sigma_{w}^{4}
\end{aligned}
$$

where $A_{M}(\varphi)$ is defined in (26). Finally, taking (77) and (80) into account, yields the MSEE of $\hat{\varphi}_{C M L}$ as expressed in (25).

\section{APPENDIX B}

In this Appendix we solve the optimization problem (47), which is reformulated as

$$
\begin{gathered}
\min _{\tilde{\varphi}}\left\{\min _{\tilde{\boldsymbol{\theta}}} \sum_{p=0}^{P-1}\left\|\mathbf{x}(p)-\mathbf{A}_{2}(\tilde{\varphi}) \tilde{\boldsymbol{\theta}}(p)\right\|^{2}\right\} \\
\text { s.t. }\|\tilde{\mathbf{b}}\|^{2} \leq \delta\|\tilde{\mathbf{a}}\|^{2}
\end{gathered}
$$

We start by solving the inner optimization problem with respect to $\tilde{\theta}$ and for a fixed $\tilde{\varphi}$. Applying the Karush-KuhnTucker (KKT) conditions to the Lagrangian function

$\mathcal{L}(\tilde{\mathbf{a}}, \tilde{\mathbf{b}}, \lambda)=\sum_{p=0}^{P-1}\|\mathbf{x}(p)-\tilde{a}(p) \mathbf{u}(\tilde{\varphi})-\tilde{b}(p) \mathbf{u}(-\tilde{\varphi})\|^{2}+\lambda\left(\|\tilde{\mathbf{b}}\|^{2}-\delta\|\tilde{\mathbf{a}}\|^{2}\right)$

we obtain

$$
\begin{gathered}
\frac{\partial}{\partial \tilde{a}^{*}(p)} \mathcal{L}(\tilde{\mathbf{a}}, \tilde{\mathbf{b}}, \lambda)=\left[\|\mathbf{u}(\tilde{\varphi})\|^{2}-\lambda \delta\right] \tilde{a}(p) \\
+\mathbf{u}^{H}(\tilde{\varphi}) \mathbf{u}(-\tilde{\varphi}) \tilde{b}(p)-\mathbf{u}^{H}(\tilde{\varphi}) \mathbf{x}(p)=0 \\
\frac{\partial}{\partial \tilde{b}^{*}(p)} \mathcal{L}(\tilde{\mathbf{a}}, \tilde{\mathbf{b}}, \lambda)=\mathbf{u}^{H}(-\tilde{\varphi}) \mathbf{u}(\tilde{\varphi}) \tilde{a}(p) \\
+\left[\|\mathbf{u}(-\tilde{\varphi})\|^{2}+\lambda\right] \tilde{b}(p)-\mathbf{u}^{H}(-\tilde{\varphi}) \mathbf{x}(p)=0
\end{gathered}
$$

for $p=0,1, \ldots, P-1$, with

$$
\lambda \geq 0 \quad\|\tilde{\mathbf{b}}\|^{2}-\delta\|\tilde{\mathbf{a}}\|^{2} \leq 0 \quad \lambda\left(\|\tilde{\mathbf{b}}\|^{2}-\delta\|\tilde{\mathbf{a}}\|^{2}\right)=0 .
$$

After some algebraic computations, the solution of the KKT equations is found to be

$$
\begin{aligned}
\hat{a}(p) & =\frac{[M+\lambda(\tilde{\varphi})] \mathbf{u}^{H}(\tilde{\varphi}) \mathbf{x}(p)-\mathbf{u}^{H}(\tilde{\varphi}) \mathbf{u}(-\tilde{\varphi}) \mathbf{u}^{H}(-\tilde{\varphi}) \mathbf{x}(p)}{[M-\delta \lambda(\tilde{\varphi})][M+\lambda(\tilde{\varphi})]-\left|\mathbf{u}^{H}(\tilde{\varphi}) \mathbf{u}(-\tilde{\varphi})\right|^{2}} \\
\hat{b}(p) & =\frac{[M-\delta \lambda(\tilde{\varphi})] \mathbf{u}^{H}(-\tilde{\varphi}) \mathbf{x}(p)-\mathbf{u}^{H}(-\tilde{\varphi}) \mathbf{u}(\tilde{\varphi}) \mathbf{u}^{H}(\tilde{\varphi}) \mathbf{x}(p)}{[M-\delta \lambda(\tilde{\varphi})][M+\lambda(\tilde{\varphi})]-\left|\mathbf{u}^{H}(\tilde{\varphi}) \mathbf{u}(-\tilde{\varphi})\right|^{2}}(84 \mathrm{~b}) \\
\lambda(\tilde{\varphi}) & =\max \left(0, \frac{\Upsilon_{2}(\tilde{\varphi})-\sqrt{\Upsilon_{2}^{2}(\tilde{\varphi})-\Upsilon_{1}(\tilde{\varphi}) \Upsilon_{3}(\tilde{\varphi})}}{\Upsilon_{1}(\tilde{\varphi})}\right)^{84 \mathrm{c})}
\end{aligned}
$$

where $\Upsilon_{1}(\tilde{\varphi}), \Upsilon_{2}(\tilde{\varphi})$ and $\Upsilon_{3}(\tilde{\varphi})$ are defined in (55)-(57). The optimal value of $\tilde{\varphi}$ that solves (81) is eventually obtained by searching for the global minimum of the concentrated likelihood function, yielding

$$
\hat{\varphi}_{c}=\arg \min _{\tilde{\varphi} \in[-\pi, \pi)} \sum_{p=0}^{P-1}\|\mathbf{x}(p)-\hat{a}(p) \mathbf{u}(\tilde{\varphi})-\hat{b}(p) \mathbf{u}(-\tilde{\varphi})\|^{2} .
$$

$n$ Taking (84a) and (84b) into account, after some computations we obtain the CJML estimator shown in (48)-(50).

\section{APPENDIX C}

In this Appendix we compute the CRB for the estimation of $\varphi$ based on the signal model shown in (63) and (64). For this purpose, we collect the unknown parameters into a $(4 P+1)$ dimensional vector $\varsigma=\left[\varphi \mathbf{z}^{T}\right]^{T}$ and let $\mathbf{C}_{w}$ be the correlation 
matrix of $w$ in (63). Then, the entries of the Fisher information matrix (FIM) $\mathbf{F}_{\boldsymbol{\varsigma}}$ are given by [21]

$$
\left[\mathbf{F}_{\boldsymbol{\varsigma}}\right]_{k_{1}, k_{2}}=\left(\frac{\partial \boldsymbol{\eta}}{\partial \varsigma_{k_{1}}}\right)^{T} \mathbf{C}_{w}^{-1}\left(\frac{\partial \boldsymbol{\eta}}{\partial \varsigma_{k_{2}}}\right) \quad 1 \leq k_{1}, k_{2} \leq 4 P+1 .
$$

Taking (65)-(67) into account, after lengthy computations we get

$$
\mathbf{F}_{\boldsymbol{\varsigma}}=\left[\begin{array}{cc}
\gamma & \mathbf{m}^{T} \\
\mathbf{m} & \mathbf{M}
\end{array}\right]
$$

where $\gamma=\mathbf{z}^{T} \dot{\mathbf{Q}}^{T} \mathbf{C}_{w}^{-1} \dot{\mathbf{Q}} \mathbf{z}, \mathbf{m}=\mathbf{Q}^{T} \mathbf{C}_{w}^{-1} \dot{\mathbf{Q}} \mathbf{z}$ and $\mathbf{M}=$ $\mathbf{Q}^{T} \mathbf{C}_{w}^{-1} \mathbf{Q}$. In the latter expressions, $\mathbf{Q}$ is defined as

$$
\dot{\mathbf{Q}}=\frac{\partial \mathbf{Q}}{\partial \varphi}=\left[\begin{array}{llll}
\dot{\mathbf{Q}}_{0}^{T} & \dot{\mathbf{Q}}_{1}^{T} & \cdots & \dot{\mathbf{Q}}_{M-1}^{T}
\end{array}\right]^{T}
$$

with $\dot{\mathbf{Q}}_{m}=\operatorname{diag}\{\underbrace{\dot{\mathbf{R}}_{m}, \dot{\mathbf{R}}_{m}, \ldots, \dot{\mathbf{R}}_{m}}_{P}\}$ and

$\dot{\mathbf{R}}_{m}=\left(m-\frac{M-1}{2}\right)\left[\begin{array}{ccc}-\mathrm{s}_{m}(\varphi) & -\mathrm{c}_{m}(\varphi) & -\mathrm{s}_{m}(\varphi) \\ \mathrm{c}_{m}(\varphi) & -\mathrm{s}_{m}(\varphi) & -\mathrm{c}_{m}(\varphi)\end{array}\right.$

The CRB for the estimation of $\varphi$ corresponds to $\left[\mathbf{F}_{\boldsymbol{\varsigma}}^{-1}\right]_{1,1}$. Using well-known results for the inverse of a partitioned matrix [21], we obtain

$$
\operatorname{CRB}(\varphi)=\frac{1}{\gamma-\mathbf{m}^{T} \mathbf{M}^{-1} \mathbf{m}}
$$

which reduces to (68) after using the expressions of $\gamma, \mathbf{m}$ and M.

\section{REFERENCES}

[1] W. Namgoong and T. H. Meng, "Direct-conversion RF receiver design", IEEE Trans. on Commun., vol. 49, n. 3, pp. 518-529, Mar. 2001.

[2] F. Yan, W.-P. Zhu, and M. O. Ahmad, "Carrier frequency offset estimation for OFDM systems with I/Q imbalance", in Proc. of 47th IEEE Midwest Symposium on Circuits and Systems, MWSCAS '04, vol. 2, pp. 633-636, July 2004

[3] L. Lanante, Jr., M. M. Kurosaki, and H. Ochi, "Low complexity compensation of frequency dependent I/Q imbalance and carrier frequency offset for direct conversion receivers", in Proc. of IEEE Inter. Symp. on Circuits and Systems (ISCAS) 2010, pp. 2067-2070, June 2010.

[4] E. L.-Estraviz, S. De Rore, F. Horlin, and L. Van der Perre, "Joint estimation of carrier frequency offset and IQ imbalance for 4G mobile wireless systems", in Proc. of Int. Conf. on Commun. (ICC2006), vol. 5, pp. 2066-2071, June 2006.

[5] Y.-C. Pan and S.-M. Phoong, "A new algorithm for carrier frequency offset estimation in the presence of I/Q imbalance", in Proc. of IEEE Vehic. Techn. Conf. (VTC 2010-Spring), pp. 1-5, Apr. 2010.

[6] M. Morelli and M. Moretti, "Carrier frequency offset estimation for OFDM direct-conversion receivers", IEEE Trans. on Wireless Commun., vol. 11, n. 7, pp. 2670-2679, Jul. 2012.

[7] G. Xing, M. Shen, and H. Liu, "Frequency offset and I/Q imbalance compensation for direct-conversion receivers", IEEE Trans. on Wireless Commun., vol. 4, pp. 673-680, March 2005.

[8] Y.-C. Pan and S.-M. Phoong, "A time-domain joint estimation algorithm for CFO and I/Q imbalance in wideband direct-conversion receivers", IEEE Trans. on Wireless Commun., vol. 11, n. 7, pp. 2353-2361, July 2012.

[9] X. Wang, Y. Xue, L. Liu, F. Ye and J. Ren, "Carrier frequency offset estimation in the presence of I/Q mismatch for wideband OFDM systems", in Proc. of IEEE 55th Int. Symp. on Circuits and Systems (MWSCAS), pp. 924-927, 2012.

[10] R. Kume, H. Lin and K. Yamashita, "Repeated preamble based carrier frequency offset estimation in the presence of I/Q imbalance", in Proc. of IEEE Int. Conf. on Commun. (ICC 2012), pp. 4867-4871, 2012.
[11] M. Morelli, M. Moretti, and H. Lin, "ESPRIT-based carrier frequency offset estimation for OFDM direct-conversion receivers", IEEE Commun. Letters, vol. 17, n. 8, pp. 1513-1516, Aug. 2013.

[12] M. Morelli and M. Moretti, "A SAGE approach to frequency recovery in OFDM direct-conversion receivers", IEEE Commun. Letters, vol. 18, n. 4, pp. 536-539, Apr. 2014.

[13] U. Tureli, H. Liu and M. Zoltowski, "OFDM blind carrier offset estimation: ESPRIT", IEEE Trans. Commun., vol. 48, n. 9, pp. 14591461, Sept. 2000.

[14] J. A. Fessler and A. O. Hero, "Space-alternating generalized expectationmaximization algorithm", IEEE Trans. on Signal Processing, vol. 42, n. 10, pp. 2664-2677, Oct. 1994.

[15] M. Ghogho, A. Swami, and P. Ciblat, "Training design for CFO estimation in OFDM over correlated multipath fading channels", in Proc. of Global Telecommun. Conf. (GLOBECOM) 2007, pp 2821-2825, 2007.

[16] M. Valkama, M. Renfors, and V. Koivunen, "Advanced methods for I/Q imbalance compensation in communication receivers," IEEE Trans. on Signal Processing, vol. 49, n. 10, pp. 2335-2344, Oct. 2001.

[17] Wireless LAN medium access control (MAC) and physical layer (PHY) specifications, higher speed physical layer extension in the $5 \mathrm{GHz}$ band. IEEE 802.11 WG, Sep. 1999, Supplement to IEEE 802.11 Standard.

[18] T. M. Schmidl and D. C. Cox, "Robust frequency and timing synchronization for OFDM", IEEE Trans. on Commun., vol. 45, n. 12, pp. 1613-1621, Dec. 1997.

( $\varphi$ (1) 9] M. Morelli and U. Mengali, "An improved frequency offset estimator for OFDM applications", IEEE Commun. Letters, vol. 3, n. 3, pp. 75-77, Mar. 1999.

[20] G.-T. Gil, I.-H. Sohn, J.-K. Park, and Y. H. Lee, ”Joint ML estimation of carrier frequency, channel, I/Q mismatch, and DC offset in communication receivers", IEEE Trans. on Vehic. Technology, vol. 54, pp. 338-349, Jan. 2005.

[21] S. M. Kay, Fundamentals of Statistical Signal Processing: Estimation Theory, Englewood Cliffs, NJ: Prentice Hall, 1993.

[22] H. M. Meyers and L. E. Franks, "Joint carrier phase and symbol timing recovery for PAM systems", IEEE Trans. on Commun., vol. COM-28, pp. 1121-1129, Aug. 1980. 